\title{
Parental and self-reported dietary and physical activity habits in pre-school children and their socio-economic determinants
}

\author{
Mercedes Sotos-Prieto ${ }^{1,2, *}$, Gloria Santos-Beneit ${ }^{1,2}$, Stuart Pocock ${ }^{1,3}$, Juliana Redondo', \\ Valentín Fuster ${ }^{1,4}$ and José L Peñalvo ${ }^{1}$ \\ 'Department of Epidemiology, Atherothrombosis and Imaging, Centro Nacional de Investigaciones \\ Cardiovasculares (CNIC), 28029 Madrid, Spain: ${ }^{2}$ International SHE Foundation, Barcelona, Spain: ${ }^{3}$ Department of \\ Medical Statistics, London School of Hygiene and Tropical Medicine, London, UK: ${ }^{4}$ Department of Cardiology, \\ Mount Sinai School of Medicine, New York, NY, USA
}

Submitted 21 August 2013: Final revision received 11 February 2014: Accepted 14 February 2014: First published online 3 April 2014

\begin{abstract}
Objective: To assess the agreement between self-reported and parent-reported dietary and physical activity habits in children; and to evaluate the socio-economic determinants of healthier habits (Mediterranean diet and physical activity) among children.

Design: Cross-sectional analysis of children recruited to a cluster-randomized controlled trial (Program SI!). Information about children's and parents' dietary and physical activity habits was obtained through validated questionnaires (Program SI! questionnaires, Kidmed, Krece Plus and Predimed scores).

Setting: Twenty-four schools in Madrid, Spain.

Subjects: Children ( $n$ 2062) aged 3-5 years and their parents ( $n$ 1949).

Results: There was positive agreement between parental- and self-reporting for three of the six children's habits examined. Parents' dietary and physical activity patterns were associated with those of their children. The main determinants of higher scores in children were higher parental age, the mother's scores, Spanish origin and higher awareness of human health $(P<0 \cdot 005)$. Children from parents with a low educational level had lower odds for scoring positively on items such as using olive oil $(\mathrm{OR}=0.23 ; 95 \% \mathrm{CI} 0.13,0.41)$ and not skipping breakfast (OR $=0.36 ; 95 \% \mathrm{CI} 0.23,0.55)$, but higher odds for meeting the recommendations for consuming pulses (OR $=1 \cdot 71 ; 95 \%$ CI 1·14, 2.55). Other habits being influenced by parental socio-economic status included the consumption of vegetables, fish, nuts, avoidance of fast food, and consumption of bakery products for breakfast. Conclusions: Children's habits may be influenced by their parents' health awareness and other socio-economic characteristics. These findings suggest that intervention strategies, even in very young children, should also target parents in order to achieve maximum success.
\end{abstract}

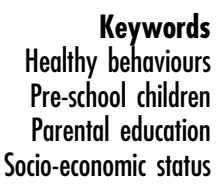

The acquisition of healthy habits, especially those related to diet and physical activity, are greatly influenced by one's environment, including political, cultural, social, demographic and economic factors ${ }^{(1,2)}$. Education plays a fundamental role in the acquisition and development of knowledge about healthy behaviours, that will develop into healthy attitudes and therefore healthy habits ${ }^{(3)}$. This is especially important during early childhood, when behaviours are formed largely through the influence of the child's immediate environment, which is mostly the family $^{(2,4)}$. Knowledge about basic nutrition is, for instance, one of the key influences on eating behaviours and therefore has been the target of many intervention strategies and policies ${ }^{(5)}$. However, unhealthy behaviours are influenced only weakly by interventions that focus on a single factor and overall improvements in healthy lifestyle require more comprehensive interventions into the individual's attitudes and habits. One of the best dietary patterns to follow given the scientific evidence supporting its cardioprotective effects is the Mediterranean diet ${ }^{(6,7)}$.

Parents are usually the principal role model for their children and there is a strong parent-child association in terms of dietary habits ${ }^{(8-11)}$. However, a recent systematic review aimed at defining the degree of association between the dietary intake of children and that of their parents showed that the agreement is weak and varies considerably between studies ${ }^{(12)}$. Dietary assessment in young children is challenging and most of the few studies conducted were based on parental reporting of their children's habits ${ }^{(12)}$. There are concerns about whether parental responses reflect accurately children's behaviour or represent only an idealized view. Few studies have 
addressed this issue and analysed whether there is a genuine association between the dietary and physical activity habits of parents and their children, and even fewer studies have been conducted with children under 5 years of age ${ }^{(4,13-15)}$.

Parental sociodemographic factors such as ethnicity or literacy seem to influence the ability of children to opt for healthy choices ${ }^{(1,16)}$. Many studies have shown that a low educational level in parents is linked to less healthy dietary habits in their children and to unhealthy behaviours in general $^{(16-19)}$. The design of effective strategies for health promotion therefore requires an understanding of the determinants of healthy choices in children from a very early age.

The present study evaluated the dietary and physical activity habits of Spanish children aged 3-5 years, with the following specific aims: (i) to evaluate whether self-reported dietary and physical activity habits of children correspond to the assessment of these habits by the children's parents; (ii) to study whether parents' health-related behaviours and other characteristics are associated with those of their children; and (iii) to explore how children's dietary and physical activity habits are influenced by parental educational level and income status.

\section{Methods}

\section{Study design and population}

Program SI! is an educational school intervention aimed at promoting cardiovascular health through the acquisition of healthy behaviours from early childhood. The efficacy of this intervention is currently being assessed through a cluster-randomized controlled trial of children aged 3-5 years and their parents, teachers and school environment, carried out in the city of Madrid, Spain, in a total of twenty-four schools (ClinicalTrials registration number: NCT01579708). With regard to the recruitment of schools, the trial is based on a hierarchical design where schools are the experimental units that receive the intervention and therefore are the units that are randomized to either intervention or control group. We randomized the schools using the percentage of immigration as a stratification factor with four levels (according to quartiles) to ensure that the groups were balanced with respect to differences in cultural background. From the total of seventy-three schools that were invited to participate, thirty-five agreed to participate and finally twenty-four were chosen, excluding larger schools with more than two classrooms per level in order to have a similar number of students in each school. The primary outcome of the trial is the 3-year change in markers of behavioural change, defined as knowledge, attitudes and habits (KAH) in four areas: (i) healthy diet; (ii) improved physical activity patterns; (iii) knowledge of the human body and heart; and (iv) management of emotions. The Program SI! is implemented in the classroom during four complete weeks within each of the three academic years (from 2011 to 2014). The detailed study protocol and the materials and strategies used for the intervention have been described elsewhere ${ }^{(20)}$. After a school year follow-up, results have recently been published showing changes in scores for KAH among children ${ }^{(21)}$.

For the present study we used baseline data collected during October to November 2011 from children (3-5 years old, $n$ 2062; 1021 girls, 1041 boys) and their parents ( $n$ 1949; 1519 women, 430 men). A cross-sectional design was adopted for the purpose of the present study.

The study was conducted in accordance with the Declaration of Helsinki and all procedures involving participants were approved by the Regional Committee for Clinical Research Ethics (CEIC-R) for the Madrid area. Informed written consent was required from the children's parents or legal guardians. The document specifies the purpose of the intervention and evaluation study, as well as the various tests and measurements that are taken. Once parents or guardians had signed the informed consent, they and their child or children were formally included in the study. A data encryption system was used to guarantee confidentiality of the information provided. The information collected is managed according to Spanish Law 15/1999 for the Protection of Personal Data.

\section{Questionnaires}

Children's dietary and physical activity assessment Information about children's habits was obtained through the Program SI! questionnaire, which assesses KAH in relation to diet and physical activity ${ }^{(22,23)}$ (Table 1 ).

Table 1 Children's and parents' questionnaires used in Program SI! (including the number of items, score range and outcome evaluated)

\begin{tabular}{|c|c|c|c|c|c|c|c|}
\hline Target population & Questionnaire & Reporting & $\begin{array}{l}\text { Behavioural } \\
\text { component }\end{array}$ & $\begin{array}{l}\text { No. of } \\
\text { items }\end{array}$ & Range & Outcome & Reference \\
\hline \multirow[t]{2}{*}{ Children } & SI!-Children & Self-report & $\begin{array}{l}\text { Diet } \\
\text { PA }\end{array}$ & $\begin{array}{l}4 \\
2\end{array}$ & $\begin{array}{l}0-4 \\
0-2\end{array}$ & $\begin{array}{l}\text { Habits } \\
\text { Habits }\end{array}$ & Cespedes et al. \\
\hline & $\begin{array}{l}\text { Kidmed } \\
\text { Krece Plus }\end{array}$ & $\begin{array}{l}\text { Parental report } \\
\text { Parental report }\end{array}$ & $\begin{array}{l}\text { Diet } \\
\text { PA }\end{array}$ & $\begin{array}{r}2 \\
16 \\
2\end{array}$ & $\begin{array}{l}-4 \text { to } 12 \\
0-10\end{array}$ & $\begin{array}{l}\text { Adherence to MD } \\
\text { Habit }\end{array}$ & Serra-Majem et al. ${ }^{(24)}$ \\
\hline \multirow[t]{2}{*}{ Parents } & SI!-Parents & Self-report & $\begin{array}{l}\text { Diet } \\
\text { PA } \\
\text { Health }\end{array}$ & $\begin{array}{r}12 \\
11 \\
5\end{array}$ & $\begin{array}{l}0-30 \\
0-30 \\
0-20\end{array}$ & $\begin{array}{l}\mathrm{KAH} \\
\mathrm{KAH} \\
\mathrm{KA}\end{array}$ & Cespedes et al. ${ }^{(23)}$ \\
\hline & Predimed & Self-report & Diet & 14 & $0-14$ & Adherence to MD & Schröder et al. (25) \\
\hline
\end{tabular}

PA, physical activity; K, knowledge; A, attitudes; $\mathrm{H}$, habits; MD, Mediterranean diet. 
This information was self-reported by the children with guided assistance by a trained psychologist. Additionally, parents completed two short questionnaires previously developed for the Spanish enKid study ${ }^{(24)}$ to evaluate children's adherence to a Mediterranean diet (Kidmed) and physical activity habits (Krece Plus; Table 1).

The Kidmed questionnaire consists of sixteen questions related to the principles of Mediterranean dietary patterns. The score ranges from -4 to 12 points, since questions with negative connotations with respect to the Mediterranean diet are assigned a value of -1 (frequent intake of fast food, increased consumption of sweets, skipping breakfast, frequent intake of pastries for breakfast). Parameters with positive connotations are assigned +1 point (e.g. takes a fruit or fruit juice every day, consumes fish regularly (at least 2-3 times/week)) as indicated previously ${ }^{(24)}$. As suggested by Serra-Majem et al. ${ }^{(24)}$, the total score was divided into three categories of Mediterranean diet quality: $\leq 3$ points, poor diet quality; $4-7$ points, average diet quality; and $\geq 8$ points, good diet quality (optimal Mediterranean diet style).

The Krece Plus score ranges from 0 to 10 points and classifies lifestyle according to the average daily hours watching television or playing video games and the average weekly hours engaged in after-school physical activity. The test scoring system classifies children's lifestyle as poor ( $\leq 5$ points for boys, $\leq 4$ points for girls), average (6-8 points for boys, 5-7 points for girls) or good ( $\geq 9$ points for boys, $\geq 8$ points for girls).

\section{Parents' dietary and physical activity assessment}

Parents completed a Program SI! questionnaire ${ }^{(20,23)}$ that assessed KAH related to diet, physical activity and knowledge of human health (Table 1). Physical activity habits were assessed as the number of days during the past week on which respondents engaged in physical activity for at least $30 \mathrm{~min}$ and the hours per workday spent seated while engaged in non-work related activities (Table 1).

Additionally, parents also completed a validated fourteenitem questionnaire (Predimed) assessing adherence to the traditional Mediterranean diet ${ }^{(25)}$. Values of 0 or 1 were assigned to each questionnaire item, producing a test score between 0 and 14 points, with higher scores indicating greater Mediterranean diet adherence ${ }^{(25)}$. Scores were grouped to define three categories: poor adherence to the Mediterranean diet ( $\leq 4$ points), average adherence (5-8 points) and high adherence (good: $\geq 8$ points).

\section{Cross-referenced items and agreement}

In the assessment of children's self-reported habits, four items for dietary habits and two for physical activity were identical in both the Program SI! and enKid (Kidmed and Krece Plus) questionnaires. The duplicated dietary items relate to questions on breakfast habits (skipping breakfast, eating dairy products, eating cereals or grains, eating commercially baked goods or pastries) and the duplicated physical activity items relate to sedentary behaviour.

For the comparison of children and parents, five items were common to the Kidmed and Predimed questionnaires: vegetable consumption ( $\geq 2$ servings/d), fruit consumption ( $\geq 2$ servings/d), fish consumption ( $>2-3$ servings/week), nut consumption ( $>2-3$ servings/week) and use of olive oil as the main culinary fat at home. This information was used to evaluate the degree of association and the influence between parents' and children's dietary habits.

\section{Socio-economic and demographic data}

Parents completed a short questionnaire to gather information on age, gender, relationship with the child or children (mother, father or legal guardian), education level, number of persons in the household and family income. Parental education level was first classified according to the guidelines of the Instituto Nacional de Estadística (INE; www.ine.es) and then categorized into three bands according to the International Standard Classification of Education (ISCED; http://www.uis.unesco.org/Education/Pages/internationalstandard-classification-of-education.aspx, 2011): low (none or primary studies; ISDED 0-2), medium (completed high school; ISCED 3 or 4) and high (high qualification or completed university degree; ISCED 5 or 6). Income status was classified as above or below (or equal to) the Spanish annual average of $€ 22500$ (Wage Structure Survey, 2009; www.ine.es).

\section{Statistical methods}

Continuous variables are presented as means and standard deviations, whereas categorical variables are presented as absolute and relative frequencies. Categorical variables were compared by using contingency tables and the $\chi^{2}$ test. The $t$ test and ANOVA were applied to compare unadjusted means. Kappa statistics were used to evaluate the agreement between questions related to dietary and physical activity habits answered by children and their parents. We calculated Pearson correlation coefficients and kappa statistics to measure the association between parents' healthy habits and those of their children.

We conducted a nested analysis adjusting for school level, as children from the same schools may be more similar to each other. Logistic regression analyses were used to examine the effect of parental education level and income status on the percentage of respondents achieving a 1-point positive score for each healthy behaviour of the questionnaires. Dichotomous variables were used for all the questionnaire items assessed. High parental education level or high income status was set as the reference category. The influence of participants' sociodemographic characteristics on Kidmed and Krece Plus scores was assessed by ANCOVA models. All models were adjusted for gender (male, female), age (as continuous), origin (Madrid, rest of Spain, rest of Europe, Latin America, Africa, others) and school plus education level when this 
Table 2 Baseline characteristics of the study sample: children ( $n$ 2062) aged 3-5 years and their parents $(n$ 1949), Madrid, Spain, October-November 2011 (Program SI!)

\begin{tabular}{|c|c|c|c|c|}
\hline & $n$ & $\%$ & Mean & SD \\
\hline Schools & 24 & & & \\
\hline Children & 2062 & & & \\
\hline Age (years) & 2062 & & 3.73 & 0.93 \\
\hline Gender (girls) & 1021 & 49.5 & & \\
\hline \multicolumn{5}{|l|}{ School year } \\
\hline Age 3 & 826 & $40 \cdot 1$ & & \\
\hline Age 4 & 640 & 31.0 & & \\
\hline Age 5 & 596 & 28.9 & & \\
\hline Kidmed score (range -4 to 12 points) & 2062 & & $7 \cdot 38$ & 2.04 \\
\hline Krece Plus score (range $0-10$ points) & 2062 & & 5.48 & 1.98 \\
\hline Parents & 1949 & & & \\
\hline Age (years) & & & 36.45 & $5 \cdot 84$ \\
\hline Female & 1519 & $77 \cdot 9$ & & \\
\hline \multicolumn{5}{|l|}{ Income level } \\
\hline$>€ 22500$ & 650 & $36 \cdot 5$ & & \\
\hline$\leq € 22500$ & 1130 & 63.5 & & \\
\hline \multicolumn{5}{|l|}{ Education level } \\
\hline High & 1197 & $63 \cdot 3$ & & \\
\hline Medium & 389 & $20 \cdot 6$ & & \\
\hline Low & 304 & $16 \cdot 1$ & & \\
\hline Predimed score (range 0-14 points) & 1949 & & $6 \cdot 31$ & $2 \cdot 40$ \\
\hline
\end{tabular}

Continuous variables are expressed as mean and standard deviation; categorical variables as $n$ (sample size) and percentage.

was not the variable of interest. The $P$ value for linear trend was tested across each category.

Statistical significance was set at the $<0.05$ level and all tests were two-tailed. All statistical analyses were conducted with the STATA statistical software package version $12 \cdot 0$.

\section{Results}

\section{Sample characteristics}

A total of 2062 children (49.5\% girls) and 1949 parents ( $77.9 \%$ women) were involved in the study at baseline (Table 2). The mean age of children was 3.7 (SD 0.9) years and that of parents was 36.5 (sD 5.8) years. Most parents self-reported medium (20.6\%) or high (63.3\%) education level, and $36.5 \%$ of parents reported an annual salary above €22500. Diet quality within the household, measured by adherence to a Mediterranean diet pattern, was moderate for both children (average Kidmed score $=7.4$ (SD 2.0) points) and parents (average Predimed score $=6 \cdot 3$ (sD 2.4) points). Children's scores on physical activity habits were also moderate (Krece Plus score $=5 \cdot 5$ (SD 2.0) points).

\section{Dietary and physical activity reporting of children's habits by parents and children}

The level of agreement for items common to parent- and self-reported assessments of children's habits is shown in Table 3. Reporting was in agreement for item 1 (skips breakfast $)$ and across age strata $(\kappa>0 \cdot 1 ; \quad P<0.001$; Table 3). For item 3 (cereals and grains) the overall number of agreement (yes or no) was low (1094 of 2062;
$53 \cdot 1 \%)$ but still statistically significant $\quad(\kappa=0.054$; $P=0.058)$; however, significant agreement across ages was maintained only for 5 -year-olds $(\kappa=0.091 ; P=0.020)$, and this was also the case for item 2 for 5 -year-olds ( $\kappa=0 \cdot 102 ; P=0 \cdot 007$; data not shown in Table 3 ).

Parent- and self-reported assessments diverged significantly for item 4 (commercially baked goods; $45.4 \%$ overall agreement; $\kappa=-0.058 ; P=0.001$ ). Disagreement was more pronounced for physical activity assessments, with only 24.9 $\%$ of parents and children agreeing on the time children dedicate during the week to sedentary activities (item 5: television and video games) and only $33.2 \%$ agreeing on the time spent engaged in physical activity (item 6).

\section{Association between parents' and children's dietary and physical activity habits}

The association between parents' and their children's dietary and physical activity habits is revealed by the data in Table 4. A positive agreement and higher odds to have the same dietary habits between parents and their children was found for consumption of vegetables, fruit, fish and olive oil (OR $>2 ; \kappa>0 \cdot 1 ; P<0 \cdot 001)$, but not for consumption of nuts $(P=0.516)$. The same pattern was observed for parents and their children for physical activity habits $(P<0 \cdot 001)$. Significant correlation was found for parents' and children's adherence to the Mediterranean diet $(r=0.356 ; P<0 \cdot 001$; data not shown).

\section{Sociodemographic determinants of Kidmed and Krece Plus scores}

A higher parental educational level and income status were associated significantly with higher Kidmed (mean score for educational level: $7 \cdot 59 ; 95 \%$ CI 7.47, $7 \cdot 70$ for high 
Table 3 Correspondence between parental reporting $v$. children's self-reporting of dietary and physical activity habits; children ( $n$ 2062) aged 3-5 years and their parents ( $n$ 1949), Madrid, Spain, October-November 2011 (Program SI!)

\begin{tabular}{|c|c|c|c|c|c|c|c|c|c|}
\hline \multirow[b]{3}{*}{ Item } & \multirow[b]{3}{*}{ Parental reporting (diet) $\dagger$} & \multicolumn{6}{|c|}{ Children's self-reporting (SI!-Children) } & \multirow[b]{3}{*}{$\kappa$} & \multirow[b]{3}{*}{$P$} \\
\hline & & \multicolumn{2}{|c|}{ Yes } & \multicolumn{2}{|c|}{ No } & & & & \\
\hline & & $n$ & $\%$ & $n$ & $\%$ & & & & \\
\hline \multirow[t]{3}{*}{1.} & Skips breakfast & & & & & & & 0.122 & $<0.001$ \\
\hline & Yes & 1742 & $84 \cdot 5$ & 130 & $6 \cdot 3$ & & & & \\
\hline & No & 155 & 7.5 & 35 & 1.7 & & & & \\
\hline \multirow[t]{3}{*}{2.} & Has a dairy product for breakfast (yoghurt, milk, etc.) & & & & & & & 0.033 & 0.070 \\
\hline & Yes & 1655 & $80 \cdot 3$ & 303 & 14.7 & & & & \\
\hline & No & 81 & 3.9 & 23 & $1 \cdot 1$ & & & & \\
\hline \multirow[t]{3}{*}{3.} & Has cereals or grains (bread, etc.) for breakfast & & & & & & & 0.054 & 0.008 \\
\hline & Yes & 769 & $37 \cdot 3$ & 686 & $33 \cdot 3$ & & & & \\
\hline & No & 282 & 13.7 & 325 & $15 \cdot 8$ & & & & \\
\hline \multirow[t]{3}{*}{4.} & Has commercially baked goods or pastries for breakfast & & & & & & & -0.058 & 0.001 \\
\hline & $\begin{array}{l}\text { Yes } \\
\text { No }\end{array}$ & $\begin{array}{l}216 \\
251\end{array}$ & $\begin{array}{l}10 \cdot 5 \\
12.2\end{array}$ & $\begin{array}{l}875 \\
720\end{array}$ & $\begin{array}{l}42 \cdot 4 \\
34.9\end{array}$ & & & & \\
\hline & & \multicolumn{2}{|c|}{$0-2 \mathrm{~h}$} & \multicolumn{2}{|c|}{$2-3 h$} & \multicolumn{2}{|c|}{$4-5 \mathrm{~h}$} & & \\
\hline Item & Parental reporting (physical activity) $\dagger$ & $n$ & $\%$ & $n$ & $\%$ & $n$ & $\%$ & $\kappa$ & $P$ \\
\hline \multirow[t]{4}{*}{5.} & $\begin{array}{l}\text { How many hours do you spend watching television or playing } \\
\text { video games every day? }\end{array}$ & & & & & & & -0.007 & 0.418 \\
\hline & $0-2 \mathrm{~h}$ & 28 & 1.4 & 39 & 1.9 & 6 & 0.3 & & \\
\hline & $2-3 h$ & 350 & $17 \cdot 0$ & 448 & $21 \cdot 7$ & 30 & 1.5 & & \\
\hline & $4-5 \mathrm{~h}$ & 484 & 23.5 & 640 & $31 \cdot 0$ & 37 & 1.8 & & \\
\hline \multirow[t]{4}{*}{6.} & How many hours do you spend doing physical activity per week? & & & & & & & 0.037 & 0.007 \\
\hline & $0-2 \mathrm{~h}$ & 135 & $6 \cdot 6$ & 323 & $15 \cdot 7$ & 401 & 19.5 & & \\
\hline & $2-3 \mathrm{~h}$ & 75 & $3 \cdot 6$ & 357 & $17 \cdot 3$ & 319 & 15.5 & & \\
\hline & $4-5 h$ & 56 & $2 \cdot 7$ & 201 & 9.8 & 191 & 9.3 & & \\
\hline
\end{tabular}

†Parental reporting regarding children's habits (diet: Kidmed; physical activity: Krece Plus).

Table 4 Association between parents' dietary and physical activity habits and those of their children; children ( $n$ 2062) aged 3-5 years and their parents ( $n$ 1949), Madrid, Spain, October-November 2011 (Program SI!)

\begin{tabular}{|c|c|c|c|c|c|c|c|}
\hline \multirow[b]{3}{*}{ Parents' dietary habits (Predimed) } & \multicolumn{4}{|c|}{ Children's dietary (Kidmed) and physical activity (Krece Plus) habits } & \multirow[b]{3}{*}{$\mathrm{OR} \dagger$} & \multirow[b]{3}{*}{$\kappa$} & \multirow[b]{3}{*}{$P \ddagger$} \\
\hline & \multicolumn{2}{|c|}{ Yes } & \multicolumn{2}{|c|}{ No } & & & \\
\hline & $n$ & $\%$ & $n$ & $\%$ & & & \\
\hline \multicolumn{8}{|l|}{ Vegetables (more than once daily) } \\
\hline Yes & 294 & $15 \cdot 1$ & 343 & $17 \cdot 6$ & \multirow[t]{2}{*}{$2 \cdot 84^{\star \star}$} & \multirow[t]{2}{*}{0.234} & \multirow[t]{2}{*}{$<0.001$} \\
\hline No & 304 & $15 \cdot 6$ & 1008 & 51.7 & & & \\
\hline \multicolumn{8}{|l|}{ Fruit (a second fruit every day) } \\
\hline Yes & 466 & $23 \cdot 9$ & 133 & $6 \cdot 8$ & \multirow[t]{2}{*}{$3 \cdot 16^{\star *}$} & \multirow[t]{2}{*}{0.199} & \multirow[t]{2}{*}{$<0.001$} \\
\hline No & 710 & $36 \cdot 4$ & 640 & $32 \cdot 8$ & & & \\
\hline \multicolumn{8}{|l|}{ Fish (at least 2-3 times/week) } \\
\hline Yes & 686 & $35 \cdot 2$ & 31 & 1.6 & \multirow[t]{2}{*}{$5 \cdot 47^{\star \star}$} & \multirow[t]{2}{*}{0.121} & \multirow[t]{2}{*}{$<0.001$} \\
\hline No & 988 & $50 \cdot 7$ & 244 & $12 \cdot 5$ & & & \\
\hline \multicolumn{8}{|l|}{ Nuts (at least 2-3 times/week) } \\
\hline Yes & 151 & $7 \cdot 7$ & 566 & $29 \cdot 0$ & \multirow[t]{2}{*}{0.93} & \multirow[t]{2}{*}{$-0 \cdot 14$} & \multirow[t]{2}{*}{0.516} \\
\hline No & 275 & $14 \cdot 1$ & 957 & $49 \cdot 1$ & & & \\
\hline \multicolumn{8}{|l|}{ Uses olive oil at home } \\
\hline Yes & 1726 & 88.6 & 22 & $1 \cdot 1$ & \multirow{2}{*}{$61.09^{\star *}$} & \multirow[t]{2}{*}{0.532} & \multirow{2}{*}{$<0.001$} \\
\hline \multirow[t]{2}{*}{ No } & 113 & $5 \cdot 8$ & 88 & 4.5 & & & \\
\hline & \multicolumn{2}{|c|}{ Good } & \multicolumn{2}{|c|}{ Poor + average } & \multirow[b]{2}{*}{ OR } & \multirow[b]{2}{*}{$\kappa$} & \multirow[b]{2}{*}{$P \ddagger$} \\
\hline Parents' physical activity habits & $n$ & $\%$ & $n$ & $\%$ & & & \\
\hline \multicolumn{8}{|l|}{ Physical activity } \\
\hline Good & 229 & $11 \cdot 8$ & 65 & $3 \cdot 3$ & $2 \cdot 46^{\star \star}$ & 0.128 & $<0.001$ \\
\hline Poor+ average & 171 & $8 \cdot 8$ & 1482 & $76 \cdot 1$ & & & \\
\hline
\end{tabular}

Two-sided level of significance: ${ }^{\star} P<0.05,{ }^{\star \star} P<0.001$.

TOR determined by logistic regression model. Reference category: negative answer (no).

$\ddagger P$ value for $\kappa$ test agreement. 
Kidmed

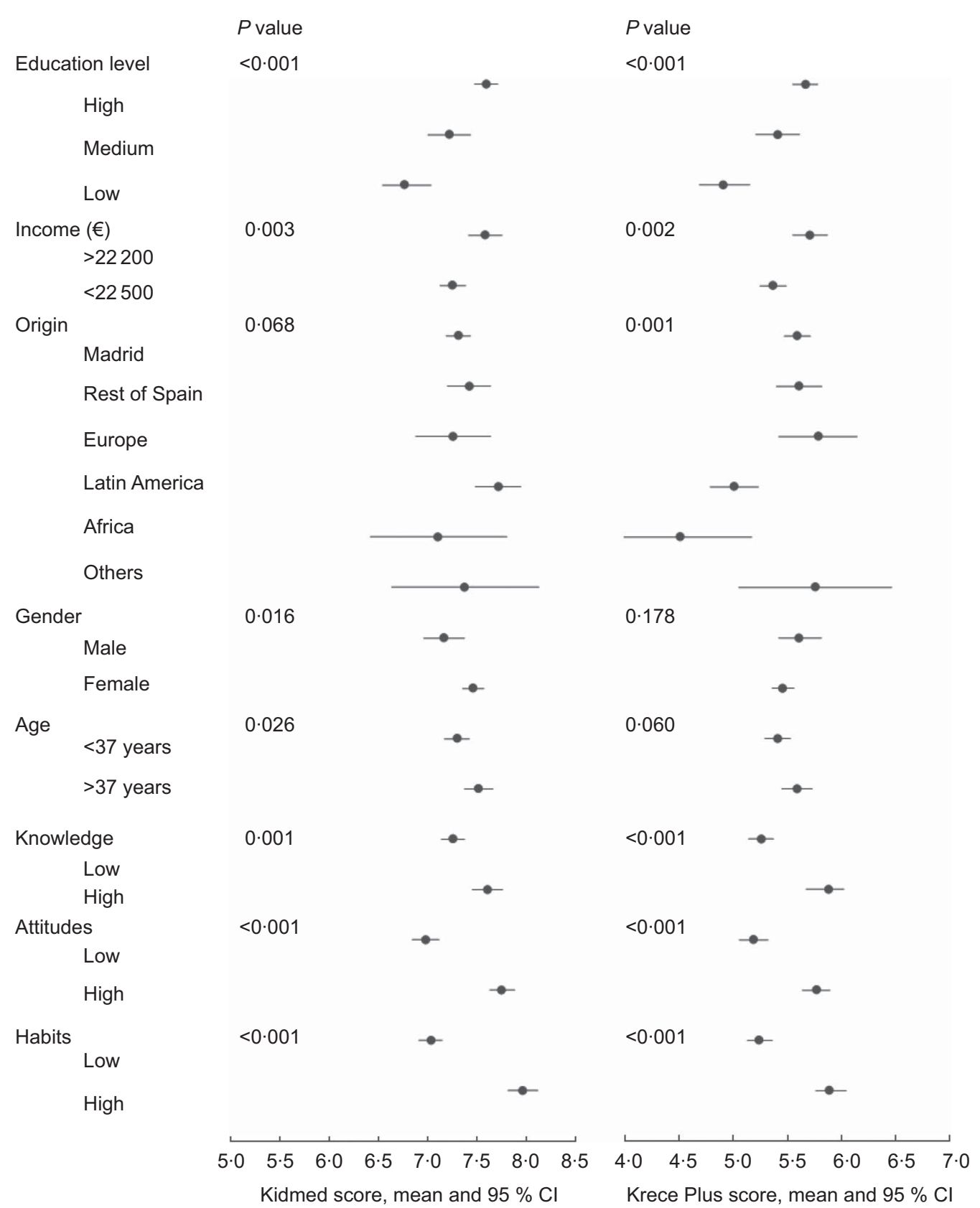

Fig. 1 Mean Kidmed and Krece Plus scores (with 95\% confidence intervals represented by horizontal bars) among children ( $n$ 2062) aged 3-5 years according to selected variables, Madrid, Spain, October-November 2011 (Program SI!). The influence of participants' sociodemographic characteristics on Kidmed scores (indicating adherence to a Mediterranean diet, range -4 to 12 points) and Krece Plus scores (indicating physical activity behaviours, range 0 to 10 points) was assessed by ANCOVA models adjusted for age, gender, geographic origin, school and socio-economic status, unless the variable was the one of interest. Statistical significance was set at the $<0.05$ level and all tests were two-sided. ' $P$ value' refers to $P$ for trend in those variables with more than two categories; otherwise, $P$ value is for the ANCOVA test

v. 6.59; $95 \%$ CI $6.56,7 \cdot 04$ for low; income status: $7 \cdot 58$; $95 \%$ CI $7 \cdot 41,7 \cdot 74$ for high $v .7 \cdot 25 ; 95 \%$ CI $7 \cdot 13,7 \cdot 38$ for low) and Krece Plus (mean score for educational level: $5 \cdot 67 ; 95 \%$ CI $5 \cdot 55,5 \cdot 78$ for high $v .4 \cdot 91 ; 95 \%$ CI $4 \cdot 69,5 \cdot 15$ for low; income status: 5.81; $95 \%$ CI 5.55, $5 \cdot 87$ for high $v$. 5.37; $95 \%$ CI 5.25, $5 \cdot 49$ for low; Fig. 1).
Parents of Latin American origin reported higher Kidmed scores $(7 \cdot 71 ; 95 \%$ CI 7.48, 7.93) but lower Krece Plus scores (5.01; $95 \%$ CI 4.79, 5.23) compared with families originating in Spain, other European countries, African or other regions. Mothers reported higher Kidmed scores (7.46; $95 \%$ CI 7.35, 7.56) than fathers $(7 \cdot 17 ; 95 \%$ CI $6.97,7 \cdot 37 ; \quad P=0 \cdot 016)$, 
whereas fathers tended to report higher scores for Krece Plus (5.61; $95 \%$ CI 5.42, 5.81) than mothers $(5 \cdot 46 ; 95 \%$ CI 5.36, $5 \cdot 56 ; P=0 \cdot 178)$. Parental age was also associated with higher scoring; compared with parents younger than 37 years, those older than 37 years reported higher scores for Kidmed (7.30; $95 \%$ CI $7 \cdot 17,7 \cdot 42 v .7 \cdot 51 ; 95 \%$ CI $7 \cdot 37,7 \cdot 65 ; P=0 \cdot 026)$ and Krece Plus (5.41; $95 \%$ CI 5.29, 5.53 v. 5.59; $95 \%$ CI 5.45, 5.73; $P=0 \cdot 060)$. Interestingly, a higher parental KAH score for healthy behaviours was significantly associated with higher Kidmed and Krece Plus scores $(P<0 \cdot 001)$.

Since socio-economic status (SES) was associated with better scores, we further investigated the influence of parental education and income status on their children's adoption of healthy habits. Frequencies of a 1-point positive score for each healthy behaviour and odds ratios (with 95\% confidence intervals) for all Kidmed and Krece Plus items are shown in Tables 5 and 6.

Parental educational level was associated with a positive answer rate frequency for children's daily consumption of fruit or juice, eating fresh or cooked vegetables daily, having cereals or grains for breakfast and having a diary product for breakfast $(P$ trend $=0.028,0.001$, $0 \cdot 004$ and $0 \cdot 015$, respectively). The largest odds ratio differences were found in the low educational level category (reference category $=$ high) for using olive oil at home $(\mathrm{OR}=0.23 ; 95 \% \mathrm{CI} 0.13,0.41)$ and skipping breakfast $(\mathrm{OR}=0.36 ; 95 \%$ CI $0.23,0.55$; Table 5). Similar results for these items were shown when analysing by income status (olive oil at home: OR=0.23; 95\% CI 0.09, 0.54; skipping breakfast: $\mathrm{OR}=0.42 ; 95 \%$ CI 0.25, 0.68; Table 6). However, higher odds of accomplishing the recommendations were observed in the low $v$. the high education category for consuming pulses (beans, peas, lentils or chickpeas) more than once weekly $(\mathrm{OR}=1.71 ; 95 \%$ CI $1.14,2.55$; Table 5).

Low parental educational level and low income status showed a similar association of lower odds of a positive score for consuming fresh cooked vegetables more than once daily and consuming fish regularly at least 2-3 times/ week ( $P$ trend $<0.005$; Tables 5 and 6 ), and going to a fast-food restaurant more than once weekly and having commercially baked foods or pastries for breakfast were associated lower odds of a positive score when comparing educational level categories (Table 5).

However, higher odds of accomplishing positive scores were observed for consuming nuts regularly $(\mathrm{OR}=1.58$; $95 \%$ CI $1.15,2.15$ for low $v$. high education level and $\mathrm{OR}=1.49 ; 95 \%$ CI 1.13, 1.97 for low $v$. high income status). We found a high percentage of pasta or rice consumption $\geq 5$ times/week $\left(\chi^{2}\right.$ test: $P=0.001$ and $\left.P<0.001\right)$ in low parental educational level and income status (Tables 5 and 6), although we did not find significant association in the logistic regression analysis.

A higher percentage of good Mediterranean diet adherence was observed in children of parents with a high educational level (53.3\%) compared with those in the middle (46.5\%) and low educational categories (42.8\%; $P<0.001)$. Similar results were found for income status (high $53.3 \% v$. low $42.8 \% ; P<0.001$ ). Similarly, a good level of physical activity was achieved by $15.0 \%$ of children whose parents had high education level compared with $13.8 \%$ of children whose parents had low education level $(P<0.001 ;$ Table 5). No significant results were found by income status $(P=0 \cdot 137)$.

\section{Discussion}

Although kappa statistics showed low rates of agreement (a paradox that has been described previously ${ }^{(26-28)}$ ), our results showed from a moderate to a positive percentage of agreement between parental assessment and children's self-reports for three of the six questions evaluated, and this association seemed to be stronger for children aged 5 years old. An inverse agreement was found for the item assessing the consumption of pastries for breakfast. While several intervention studies in children have been reported, very young children have been studied only in the IDEFICS (Identification and prevention of Dietary- and lifestyle-induced health effects In Children and infantS) study (2-9 years old) and a few others ${ }^{(4,15,16)}$. These studies assumed that parents or other child carers report children's habits accurately. Dietary assessment in children at this age is a particular challenge for investigators, since it raises the issue of the reliability of information provided by parents, who may provide, instead of an accurate assessment, what they consider to be the correct answer. We might hypothesize that the inverse agreement found in our study could be due to the fact that the parents recognize this as an unhealthy dietary habit, leading them to report low consumption for this item in contrast to the higher values reported by the children. Additional reasons could be: (i) children are accurate reporters of their own behaviour, particularly with regard to dietary intake and physical activity that may happen while at school (or at least when they are not at home under the supervision of their parents); or (ii) children are poor reporters and parents are accurate. However, this is only our interpretation and further analysis should be carried out to address this point.

Our study further provides evidence that, at least in this particular Spanish population, there is a significant association between the health habits of parents and those of their children. Parents' adherence to a Mediterranean diet and regular physical activity positively affect their children's health habits. Consistent with our results, a meta-analysis analysing parents' influence on children's eating habits showed weak or moderate correlations $(0 \cdot 20-0 \cdot 30)$ for key dietary measures ${ }^{(12)}$, although that meta-analysis included few studies with children under 5 years of age. A study of children from the ages of 2 to 18 years, using a total dietary quality score, showed a mother-daughter correlation of $0 \cdot 18$ 
Table 5 Prevalence of 1-point score and odds ratios (and $95 \%$ confidence intervals) from logistic regression, showing the correlation of parental education level with children's Kidmed and Krece Plus items; children ( $n$ 2062) aged 3-5 years and their parents ( $n$ 1949), Madrid, Spain, October-November 2011 (Program SI!)

\begin{tabular}{|c|c|c|c|c|c|c|c|c|}
\hline \multirow[b]{3}{*}{ Item } & \multicolumn{7}{|c|}{ Education level† } & \multirow[b]{3}{*}{$P$ trend } \\
\hline & \multirow{2}{*}{$\frac{\text { High }}{\%}$} & \multirow{2}{*}{$\frac{\text { Medium }}{\%}$} & \multirow{2}{*}{$\frac{\text { Low }}{\%}$} & \multicolumn{2}{|c|}{ Medium $v$. High } & \multicolumn{2}{|c|}{ Low $v$. High } & \\
\hline & & & & OR & $95 \% \mathrm{Cl}$ & OR & $95 \% \mathrm{Cl}$ & \\
\hline Takes a fruit or fruit juice every day & $94 \cdot 7$ & 94.9 & $92 \cdot 1$ & 0.94 & $0.54,1.65$ & $0.58^{*}$ & $0.35,0.98$ & 0.028 \\
\hline Has a second piece of fruit every day & $62 \cdot 3$ & $56 \cdot 8$ & $56 \cdot 2$ & 0.81 & $0.63,1.03$ & $0.76^{\star}$ & $0.59,0.99$ & 0.083 \\
\hline Has fresh or cooked vegetables regularly once per day & $77 \cdot 4$ & $75 \cdot 3$ & 68.4 & 0.82 & $0.62,1 \cdot 10$ & $0.60^{\star \star}$ & $0.44,0.80$ & 0.001 \\
\hline Has fresh or cooked vegetables more than once per day & $32 \cdot 8$ & $29 \cdot 6$ & $22 \cdot 0$ & $0.76^{*}$ & $0.58,0.99$ & $0.50^{\star \star}$ & $0.36,0.69$ & $<0.001$ \\
\hline Consumes fish regularly (at least $2-3$ times/week) & 88.9 & $81 \cdot 0$ & $81 \cdot 6$ & 0.81 & $0.57,1.15$ & $0.58^{\star *}$ & $0.39,0.84$ & 0.026 \\
\hline Goes more than once per week to a fast-food (hamburger) restaurant & $90 \cdot 8$ & $86 \cdot 6$ & 83.3 & 0.89 & $0.60,1.31$ & $0.60^{*}$ & $0.40,0.89$ & 0.012 \\
\hline Likes pulses§ and eats them more than once per week & $81 \cdot 1$ & $79 \cdot 2$ & 87.5 & 0.95 & $0.69,1.28$ & $1 \cdot 71^{\star}$ & $1.14,2.55$ & 0.003 \\
\hline Consumes pasta or rice almost every day ( $\geq 5$ times/week) & 29.5 & $38 \cdot 0$ & $37 \cdot 8$ & 1.07 & $0.81,1.41$ & 1.18 & $0.87,1.60$ & 0.231 \\
\hline Has cereals or grains (bread, etc.) for breakfast & $72 \cdot 4$ & 68.9 & $65 \cdot 1$ & 0.78 & $0.59,1.02$ & $0.69^{*}$ & $0.52,0.93$ & 0.004 \\
\hline Consumes nuts regularly (at least $2-3$ times/week) & $19 \cdot 4$ & $22 \cdot 1$ & 29.9 & 1.11 & $0.82,1.50$ & $1.58^{\star}$ & $1 \cdot 15,2 \cdot 15$ & 0.002 \\
\hline Uses olive oil at home & $97 \cdot 0$ & $91 \cdot 0$ & $88 \cdot 8$ & $0.47^{*}$ & $0.27,0.80$ & $0.23^{\star *}$ & $0.13,0.41$ & $<0.001$ \\
\hline Skips breakfast & 93.9 & $86 \cdot 9$ & $83 \cdot 6$ & $0.52^{*}$ & $0.34,0.80$ & $0.36^{\star \star}$ & $0.23,0.55$ & $<0.001$ \\
\hline Has a dairy product for breakfast (yoghurt, milk, etc.) & $96 \cdot 0$ & 94.3 & 92.8 & 0.67 & $0.39,1.17$ & $0.55^{\star}$ & $0.31,0.97$ & 0.015 \\
\hline Has commercially baked goods or pastries for breakfast & $56 \cdot 6$ & $50 \cdot 1$ & $44 \cdot 7$ & 0.82 & $0.64,1.04$ & $0.63^{\star *}$ & $0.48,0.83$ & $<0.001$ \\
\hline Takes two yoghurts and/or some cheese $(40 \mathrm{~g})$ daily & 69.5 & 71.5 & $67 \cdot 8$ & 0.95 & $0.72,1.24$ & 0.85 & $0.63,1.13$ & 0.202 \\
\hline Takes sweets and candy several times every day & 94.9 & $93 \cdot 3$ & $84 \cdot 2$ & 0.95 & $0.56,1.63$ & $0.34^{\star *}$ & $0.22,0.54$ & $<0.001$ \\
\hline Kidmed scorell & $53 \cdot 3$ & $46 \cdot 5$ & $42 \cdot 8$ & $0.59^{*}$ & $0.39,0.89$ & $0.44^{\star *}$ & $0.27,0.75$ & 0.001 \\
\hline Krece Plus scoreף & $15 \cdot 0$ & $17 \cdot 2$ & $13 \cdot 8$ & $0.72^{*}$ & $0.56,0.92$ & $0.61^{\star \star}$ & $0.46,0.80$ & $<0.001$ \\
\hline
\end{tabular}

Two-sided level of significance: ${ }^{*} P<0.05,{ }^{* *} P<0.001$.

High education level: $n$ 1197, 63.3\%; medium education level: $n$ 389, 20.6\%; low education level: $n 304,16.1 \%$.

†Adjusted $\mathrm{OR}$ and $95 \% \mathrm{Cl}$ determined by logistic regression models. Models adjusted for age, gender, geographic origin and school. Reference group $=$ high education level.

\$Pulses include beans, peas, lentils and chickpeas.

IIKidmed score (range -4 to 12 points): poor, $\leq 3$ points; average, $4-7$ points; good, $\geq 8$ points. Reference group = poor + average; OR presented for good $v$. poor + average

IKrece Plus score (range 0-10 points): poor, $\leq 5$ points (boys), $\leq 4$ points (girls); average, $6-8$ points (boys), $5-7$ points (girls); good, $\geq 9$ points (boys), $\geq 8$ points (girls). Reference group = poor + average; OR presented for good $v$. poor + average. 
Table 6 Prevalence of 1-point score and odds ratios (and 95\% confidence intervals) from logistic regression, showing the correlation of parental income status with children's Kidmed and Krece Plus items; children ( $n$ 2062) aged $3-5$ years and their parents $(n$ 1949), Madrid, Spain, October-November 2011 (Program SI!)

\begin{tabular}{|c|c|c|c|c|c|}
\hline \multirow[b]{3}{*}{ Item } & \multicolumn{4}{|c|}{ Income status $\dagger$} & \multirow[b]{3}{*}{$P$ value } \\
\hline & \multirow{2}{*}{$\frac{\text { High }}{\%}$} & \multirow{2}{*}{$\frac{\text { Low }}{\%}$} & \multicolumn{2}{|c|}{ Low v. High } & \\
\hline & & & OR $\ddagger$ & $95 \% \mathrm{Cl}$ & \\
\hline Takes a fruit or fruit juice every day & $95 \cdot 1$ & 94.3 & 0.74 & $0.46,1.20$ & 0.279 \\
\hline Has a second piece of fruit every day & 62.6 & 58.7 & 0.88 & $0.71,1.10$ & 0.249 \\
\hline Has fresh or cooked vegetables regularly once per day & $77 \cdot 1$ & $75 \cdot 0$ & 0.88 & $0.68,1.14$ & 0.384 \\
\hline Has fresh or cooked vegetables more than once per day & $32 \cdot 8$ & 29.4 & $0.74^{*}$ & $0.59,0.94$ & 0.014 \\
\hline Consumes fish regularly (at least $2-3$ times/week) & $92 \cdot 8$ & 81.9 & $0.59^{*}$ & $0.40,0.86$ & 0.003 \\
\hline Goes more than once per week to a fast-food (hamburger) restaurant & $93 \cdot 1$ & $86 \cdot 6$ & 0.72 & $0.49,1.08$ & 0.149 \\
\hline Likes pulses $\S$ and eats them more than once per week & $82 \cdot 2$ & 81.9 & 1.15 & $0.87,1.54$ & 0.446 \\
\hline Consumes pasta or rice almost every day ( $\geq 5$ times/week) & $25 \cdot 2$ & $36 \cdot 3$ & 1.07 & $0.83,1.37$ & 0.443 \\
\hline Has cereals or grains (bread, etc.) for breakfast & $71 \cdot 1$ & $69 \cdot 4$ & 0.83 & $0.66,1.05$ & $0 \cdot 170$ \\
\hline Consumes nuts regularly (at least $2-3$ times/week) & $17 \cdot 2$ & 24.1 & $1.49^{*}$ & $1.13,1.97$ & 0.011 \\
\hline Uses olive oil at home & $98 \cdot 8$ & 91.9 & $0.23^{\star \star}$ & $0.09,0.54$ & 0.002 \\
\hline Skips breakfast & $96 \cdot 0$ & 87.6 & $0.42^{\star \star}$ & $0.25,0.68$ & 0.001 \\
\hline Has a dairy product for breakfast (yoghurt, milk, etc.) & $95 \cdot 8$ & 94.6 & 0.90 & $0.53,1.54$ & 0.642 \\
\hline Has commercially baked goods or pastries for breakfast & $56 \cdot 6$ & $50 \cdot 1$ & 0.82 & $0.66,1.02$ & 0.079 \\
\hline Takes two yoghurts and/or some cheese $(40 \mathrm{~g})$ daily & $66 \cdot 5$ & $70 \cdot 6$ & 0.98 & $0.77,1.23$ & 0.937 \\
\hline Takes sweets and candy several times every day & $96 \cdot 3$ & 91.4 & $0.47^{\star}$ & $0.28,0.79$ & 0.010 \\
\hline Kidmed scorell & 52.9 & 47.7 & 0.81 & $0.65,1.01$ & 0.052 \\
\hline Krece Plus scoreף & $15 \cdot 2$ & $15 \cdot 2$ & 0.79 & $0.58,1.10$ & 0.137 \\
\hline
\end{tabular}

Two-sided level of significance: ${ }^{*} P<0.05,{ }^{*} P<0.001$

†High income status (>€22 500): $n$ 650, 36.5\%; low income status ( $\leq € 22500$ ): $n 1130,63.5 \%$.

†Adjusted OR and $95 \% \mathrm{Cl}$ determined by logistic regression models. Models adjusted for age, gender, geographic origin and school. Reference group = high income level.

§Pulses include beans, peas, lentils or chickpeas.

IIKidmed score (range -4 to 12 points): poor, $\leq 3$ points; average, $4-7$ points; good, $\geq 8$ points. Reference group = poor + average; OR presented for good $v$. poor + average.

TKrece Plus score (range $0-10$ points): poor, $\leq 5$ points (boys), $\leq 4$ points (girls); average, $6-8$ points (boys), $5-7$ points (girls); good, $\geq 9$ points (boys), $\geq 8$ points (girls). Reference group $=$ poor + average; OR presented for good $v$. poor + average.

and a mother-son correlation of $0 \cdot 28^{(29)}$. Our study shows a higher correlation $(0.356)$ and even stronger associations as the children grow. The positive association between parents' consumption of vegetables, fruit, fish and olive oil and consumption of these foods by their children has also been observed by other studies ${ }^{(30-32)}$. This suggests that parents have an important influence on their children's dietary intake and that this is especially relevant at a young age, when children develop lifelong habits.

Our findings also show variations of children's Kidmed and Krece Plus scores with specific parental characteristics. The Kidmed and Krece Plus scores were higher for children whose parents were aged 37 years or older. Moreover, Kidmed score was also higher for responses from mothers and from parents of Spanish or Latin-American origin. Our results agree with previous studies which reported that older parents included a greater number of healthy items in their children's diet ${ }^{(4)}$. This might be related to the acquisition of knowledge and awareness with age. Interestingly, we also found that parents' KAH scores for human health determined children's dietary and physical activity scores, so that children of parents with higher KAH scores are likely to have better scores for healthy behaviours. This effect was seen regardless of parents' education level, indicating that parents' KAH score for nutrition is independently associated with children's Kidmed and Krece Plus scores. In agreement with our results, an Australian study ${ }^{(33)}$ in 193 children aged 5-6 years found that parents' nutritional knowledge directly predicted that of their children, including an ability to distinguish between healthy and unhealthy foods $^{(15)}$. Studies carried out in older children (9-11 years) emphasized that better parental knowledge of nutrition leads parents to place greater emphasis on teaching their children about healthy foods and therefore to improve their health-related behaviours and choices ${ }^{(8,9,11,34)}$. The current study, with younger children, suggests that in addition to parents' knowledge, their attitudes and habits are also likely to lead to better adherence to a healthy diet and physical activity by their children.

Another factor influencing children's dietary and physical activity habits identified by our analysis is parental SES (education level and income status). Our findings indicate that this association affects most of the health-related questions evaluated. The likelihood of achieving a 1-point positive score for eating fruit or juice every day, consuming fresh or cooked vegetables daily, having cereals or grains for breakfast and having a diary product for breakfast was higher for children of parents in the highest education level category. Most previous studies that analysed parental SES found a similar relationship with health-related behaviours ${ }^{(17,18,32)}$. In the IDEFICS study ${ }^{(35)}$ on children between 2 and 9 years of age, parental educational level 
was associated with fruit and vegetable intake, as in other studies $^{(30,31)}$, but was additionally associated with intake of fish and cereals. A recent Greek study ${ }^{(19)}$ in older children (10-12-year-olds) also used the Kidmed score and found an association between higher parental education level and a healthier lifestyle and dietary profile among children (Mediterranean diet and time devoted to sports activities), expanding on earlier results obtained in another Greek population ${ }^{(36)}$.

Lower SES in our study was associated with modest scores for vegetable and fish consumption, skipping breakfast, fast-food intake and consuming pastries for breakfast. It is noteworthy that the largest differences seen in children from low-SES households were the lower olive oil consumption, the higher consumption of pulses (beans, peas, lentils or chickpeas) and the higher frequency of pasta/rice consumption, since these products are associated with purchasing power. Lower education level may be linked to lower income and subsequently to a relatively greater availability of less expensive foods such as rice, pulses and junk food ${ }^{(4)}$. Conversely, olive oil is an expensive item that is less accessible for low-income households in which cost is a key factor in food choice. Such a conclusion is consistent with a study that reported a poorer diet among 5-year-old children from less advantaged backgrounds ${ }^{(37)}$.

A strength of our study is the stratified randomization process for selecting schools for intervention, which decreases the chance of bias in the study sample. The questionnaires used to assess parents' and children's dietary and physical activity habits both yielded comparable information for the same items, thus allowing evaluation and correlation of the information obtained. In addition, many earlier studies did not include information about which parent (mother or father) ${ }^{(11,38)}$ answered the questionnaire, and our results indicate that this should be taken into account since it seems to have an influence on children's choices.

A limitation of our cross-sectional study is that, by design, bidirectionality is likely for all observed associations and cause-and-effect relationships cannot be established. Another limitation is that, since the study population is characteristic of the city of Madrid, the findings might be applicable only to similar urban environments.

\section{Conclusions}

The present study shows that children's dietary and physical activity habits may be associated with those of their parents and also influenced by the socio-economic characteristics of the household. Our results showed low agreement between parental report and children's self-report for those items that the parents recognized as representing unhealthy habits. Therefore, parental reporting bias must be taken into consideration in studies involving pre-school children, although additional reasons could be considered as well (such as children are accurate regarding their own behaviour in the school; or in contrast, children are poor reporters and parents accurate). Older parental age (>37 years), maternal health habits, Spanish origin and higher KAH in relation to human health were identified as the main parental determinants of higher scores in children. Given the difficulty of changing socio-economic indicators, interventions that include strategies to increase parental responsibility for the family's health will increase the opportunities for their children to adopt a healthier lifestyle. This intervention should begin with parents and their children in their early years, when there is a window of opportunity for instilling lifelong healthy behaviours.

\section{Acknowledgements}

Financial support: This study was funded by the Fondo de Investigación Sanitaria del Insituto de Salud Carlos III, Madrid (research grant PI11/01885). Conflict of interest: All authors declare no conflict of interest. Authorship: M.S.-P. assisted with data collection, performed data management and statistical analyses, and interpreted and drafted the manuscript. G.S.-B. coordinated data collection, interpreted results and helped draft the manuscript. R.J. interpreted data and helped draft the manuscript. S.P. oversaw statistical analyses and helped draft the manuscript. V.F. conceived the intervention study and helped draft the manuscript. J.L.P. coordinated and designed the study, interpreted the results and helped draft the manuscript. Acknowledgements: The authors would like to thank the International SHE Foundation, intellectual owner of Program SI! They also thank the ALICIA Foundation and Sesame Workshop for providing the materials needed for the intervention.

\section{References}

1. Cutler GJ, Flood A, Hannan P et al. (2011) Multiple sociodemographic and socioenvironmental characteristics are correlated with major patterns of dietary intake in adolescents. J Am Diet Assoc 111, 230-240.

2. Campbell KJ \& Hesketh KD (2007) Strategies which aim to positively impact on weight, physical activity, diet and sedentary behaviours in children from zero to five years. A systematic review of the literature. Obes Rev 8, 327-338.

3. Birch LL \& Davison KK (2001) Family environmental factors influencing the developing behavioral controls of food intake and childhood overweight. Pediatr Clin North Am 48, 893-907.

4. dos Santos Barroso G, Sichieri R \& Salles-Costa R (2012) Relationship of socio-economic factors and parental eating habits with children's food intake in a population-based study in a metropolitan area of Brazil. Public Health Nutr, (Epublication ahead of print version).

5. Worsley A (2002) Nutrition knowledge and food consumption: can nutrition knowledge change food behaviour? Asia Pac J Clin Nutr 11, Suppl. 3, S579-S585.

6. Sotos-Prieto M, Zulet MA \& Corella D (2010) Scientific evidence of the Mediterranean diet effects in determining intermediate and final cardiovascular disease phenotypes. Med Clin Barc 134, 22-29. 
7. Estruch R, Ros E, Salas-Salvado J et al. (2013) Primary prevention of cardiovascular disease with a Mediterranean diet. N Engl J Med 368, 1279-1290.

8. Mitchell GL, Farrow C, Haycraft E et al. (2013) Parental influences on children's eating behaviour and characteristics of successful parent-focussed interventions. Appetite 60, 85-94.

9. Savage JS, Fisher JO \& Birch LL (2007) Parental influence on eating behavior: conception to adolescence. J Law Med Ethics 35, 22-34.

10. Scaglioni S, Salvioni M \& Galimberti C (2008) Influence of parental attitudes in the development of children eating behaviour. Br J Nutr 99, Suppl. 1, S22-S25.

11. Lazarou C, Kalavana T \& Matalas A-L (2008) The influence of parents' dietary beliefs and behaviours on children's dietary beliefs and behaviours. The CYKIDS study. Appetite 51, 690-696.

12. Wang Y, Beydoun MA, Li J et al. (2011) Do children and their parents eat a similar diet? Resemblance in child and parental dietary intake: systematic review and metaanalysis. J Epidemiol Community Health 65, 177-189.

13. Fisher JO, Mitchell DC, Smiciklas-Wright H et al. (2002) Parental influences on young girls' fruit and vegetable, micronutrient, and fat intakes. J Am Diet Assoc 102, 58-64.

14. Longbottom PJ, Wrieden WL Pine CM (2002) Is there a relationship between the food intakes of Scottish 5(1/2)-8 (1/2)-year-olds and those of their mothers? J Hum Nutr Diet 15, 271-279.

15. Hu C, Ye D, Li Y et al. (2010) Evaluation of a kindergartenbased nutrition education intervention for pre-school children in China. Public Health Nutr 13, 253-260.

16. Garemo M, Lenner RA, Nilsson EK et al. (2007) Food choice, socio-economic characteristics and health in 4-year olds in a well-educated urban Swedish community. Clin Nutr 26, 133-140.

17. Bammann K, Gwozdz W, Lanfer A et al. (2013) Socioeconomic factors and childhood overweight in Europe: results from the multi-centre IDEFICS study. Pediatr Obes $\mathbf{8}$ $1-12$.

18. Tandon PS, Zhou C, Sallis JF et al. (2012) Home environment relationships with children's physical activity, sedentary time, and screen time by socioeconomic status. Int J Behav Nutr Phys Act 9, 88.

19. Antonogeorgos G, Panagiotakos DB, Grigoropoulou D et al. (2013) The mediating effect of parents' educational status on the association between adherence to the Mediterranean diet and childhood obesity: the PANACEA study. Int J Public Health 58, 401-408.

20. Peñalvo JL, Santos-Beneit G, Sotos-Prieto M et al. (2013) A cluster randomized trial to evaluate the efficacy of a schoolbased behavioral intervention for health promotion among children aged 3 to 5. BMC Public Health 13, 656.

21. Penalvo JL, Sotos-Prieto M, Santos-Beneit G et al. (2013) The Program SI! intervention for enhancing a healthy lifestyle in preschoolers: first results from a cluster randomized trial. BMC Public Health 13, 1208.

22. SitiSabariah B, Zalilah M, Norlijah O et al. (2006) Reliability and validity of the primary school children's nutrition knowledge, attitude and practice instrument used in the HELIC Study. Mal J Nutr 12, 33-44.

23. Cespedes J, Briceno G, Farkouh ME et al. (2013) Targeting preschool children to promote cardiovascular health: cluster randomized trial. Am J Med 126, 27-35.

24. Serra-Majem L, Ribas L, Ngo J et al. (2004) Food, youth and the Mediterranean diet in Spain. Development of KIDMED, Mediterranean Diet Quality Index in children and adolescents. Public Health Nutr 7, 931-935.

25. Schröder H, Fitó M, Estruch R et al. (2011) A short screener is valid for assessing Mediterranean diet adherence among older Spanish men and women. J Nutr 141, $1140-1145$.

26. Feinstein AR \& Cicchetti DV (1990) High agreement but low kappa: I. The problems of two paradoxes. J Clin Epidemiol 43, 543-549.

27. Viera AJ \& Garrett JM (2005) Understanding interobserver agreement: the kappa statistic. Fam Med 37, 360 -363.

28. Cicchetti DV \& Feinstein AR (1990) High agreement but low kappa: II. Resolving the paradoxes. J Clin Epidemiol 43, 551-558.

29. Beydoun MA \& Wang Y (2009) Parent-child dietary intake resemblance in the United States: evidence from a large representative survey. Soc Sci Med 68, 2137-2144.

30. Hart CN, Raynor HA, Jelalian E et al. (2010) The association of maternal food intake and infants' and toddlers' food intake. Child Care Health Dev 36, 396-403.

31. Cooke LJ, Wardle J, Gibson EL et al. (2004) Demographic, familial and trait predictors of fruit and vegetable consumption by pre-school children. Public Health Nutr 7, 295-302.

32. Riediger ND, Shooshtari S \& Moghadasian MH (2007) The influence of sociodemographic factors on patterns of fruit and vegetable consumption in Canadian adolescents. $J$ Am Diet Assoc 107, 1511-1518.

33. Zarnowiecki D, Sinn N, Petkov J et al. (2012) Parental nutrition knowledge and attitudes as predictors of 5-6-yearold children's healthy food knowledge. Public Health Nutr 15, 1284-1290.

34. Gibson EL, Wardle J \& Watts CJ (1998) Fruit and vegetable consumption, nutritional knowledge and beliefs in mothers and children. Appetite 31, 205-228.

35. Miguel Fernandez-Alvira J, Mouratidou T, Bammann K et al. (2013) Parental education and frequency of food consumption in European children: the IDEFICS study. Public Health Nutr 16, 487-498.

36. Kontogianni MD, Farmaki A-E, Vidra N et al. (2010) Associations between lifestyle patterns and body mass index in a sample of Greek children and adolescents. $J$ Am Diet Assoc 110, 215-221.

37. Skafida V (2013) The family meal panacea: exploring how different aspects of family meal occurrence, meal habits and meal enjoyment relate to young children's diets. Sociol Health Ill 35, 906-923.

38. Johannsen DL, Johannsen NM \& Specker BL (2006) Influence of parents' eating behaviors and child feeding practices on children's weight status. Obesity (Silver Spring) 14, 431-439. 